\title{
Self Adaptive Metadata Associassion and Ontology Learning
}

\author{
Prof. Mohini J. Arote ${ }^{1}$, Rupam Bhor ${ }^{2}$, Pranali Vethekar ${ }^{3}$, Amruta Pawar ${ }^{4}$ \\ Asst. Prof., JSPM's BSIOTR, Wagholi, Pune ${ }^{1}$ \\ Student, Computer, JSPM's BSIOTR, Pune, India ${ }^{2,3,4}$
}

\begin{abstract}
The internet is well documented and become the largest market in the world and online advertising is very accepted with many industries counting the customary mining service industry where mining service advertisements are effective carrier of mining repair in sequence. However, service users may encounter three major issuesHeterogeneity, Ubiquity and Ambiguity vagueness when pointed for mining check information on over the Internet. In this paper the framework of a novel self-adaptive semantic focused crawler, with the idea of exactly and efficiently discover, formatting, and indexing mining repair in sequence over the Internet, by taking into account the three major issues. The innovation of this study recline in the plan of an unconfirmed framework for vocabulary-based ontology learning, and a mixture algorithm for matching semantically pertinent concept and metadata.A series of experiments are conducted in order to assess the act of this crawler. The conclusion and the way of hope work are given in the final section.
\end{abstract}

Keyword: Mining service industry, ontology learning, semantic focused crawler, service advertisement, service information discovery.

\section{INTRODUCTION}

It is fine known that information technology has a manufacturing find out engines. Residence business thoughtful result on the mode commerce is conduct; also directory, such as Google ${ }^{\mathrm{TM}}$ home Business core and the Internet has become the major bazaar in the world. It is limited Yellowpages ${ }^{\circledR}$ Domain-specific commerce look estimated to present be above 2 billion Internet users in for engines, such as healthcare, business in addition to 2011, among an predictable yearly increase of more 16\%, sightseeing commerce look for engines explore train compare by 360 million users in 20001. Original publicity, such as Google ${ }^{\mathrm{TM}}$ and Yahoo! ${ }^{\circledR}$ publicity house. production professional contain realize the saleable These overhaul registries are geologically spread more application of the Internet equally used for their clients than the Internet.

and tactical associates, rotating the Internet into an vast shopping center by a vast catalogue. clients are intelligent to look around a enormous choice of products and service vertisements more than the Internet, and purchase these produce straight during online business system. Service advertisement figure a substantial element of the publicity which take put more the Internet and contain the following facial appearance:

a) Heterogeneity

Given the range of services in the actual earth, lots of scheme contain be future to categorize the armed forces as of a variety of perspective, counting the rights of check instrument, the property of armed forces, the personality of the service take action, release, command and provide, and thus going on. However, present is not a in public decided plan accessible used for classify examine advertisement more than Internet. in addition, even as numerous marketable item for consumption and service look for engines supply categorization scheme of armed forces with the reason of facilitate a investigate, they do not actually discriminate among the creation and the repair poster; in its place, they join together into single classification.

b) Ubiquity

Service advertisement can be register by repair provider during a variety of service registries, as well as: worldwide

\section{c) Ambiguity}

The popular of the online examine publicity in sequence is fixed in a huge quantity of in sequence on the net and is describe in usual words, thus it can be unclear. Furthermore, online repair in order do not contain a reliable set-up and normal, and vary from network side to Web page. Removal is one of the oldest industry in person the past, have emerge with the start of person society. Taking out armed forces submit to a sequence of services which hold removal, quarry, and oil and gas taking out actions. Since the start of the in order period, taking out check company include realize the authority of online publicity, and they have attempt to endorse themselves by aggressively fusion the check marketing the people. It was establish that virtually 50,000 company universal contain register their armed forces on the Kompass website. Though, these removal overhaul advertisement are also topic to the issue of heterogeneity, ubiquity and ambiguity, which stop user as of exactly and professionally pointed for removal examine in sequence more the Internet.

Examination innovation is an budding investigate locale in the domain of manufacturing informatics, which aim to mechanically or semi-automatically get back armed forces or examine in sequence in exacting environment by income of a variety of IT method. A lot of study contain been approved out in the environment of wireless network 
and dispersed manufacturing system. though, little study have been intended for engineering service poster finding in the Web surroundings, by captivating into story the heterogeneous, ubiquitous and ambiguous skin tone of examine publicity in order.

In order to contract with the over problems, in this paper, we suggest the frame of a work of fiction self-adaptive semantic focused (SASF) flatterer, by combine the technology of semantic focused crawling and ontology learning, whereby semantic focused crawling skill is use to explain the issues of heterogeneity, ubiquity and ambiguity of mining check in sequence, with ontology learn information is use to protect the high presentation of crawling in the unrestrained Web surroundings. This crawler is planned with the reason of serving investigate engines to exactly and proficiently explore removal overhaul in sequence by semantically discover, format, and indexing information.

\section{SYSTEM COMPONENTS AND WORKFLOW}

We initiate the organization design and the workflow of the future SASF crawler.
It wants to be renowned that this crawler is build ahead the semantic focused crawler planned in our earlier study. The difference among this effort and the earlier effort can be summarizing as follows:

- Our previous research work created an only semantic focused crawler, which do not have an ontologylearning function to mechanically develop the utilize ontology. This research aims to mixture this shortcoming. Our prior job utilize the examine ontology and the service metadata format, mainly calculated for the carrying service domain and the fitness care service domain. In this study, we design mining service ontology and a mining service metadata plan to crack the difficulty of self-adaptive service in sequence detection for the removal service commerce.

An impression of the scheme architecture and the workflow is shown in Fig. As can be seen, the SASF crawler consists of two information basis - a Mining Service Ontology Base and a Mining Service Metadata Base, and a series of process, as well as a workflow coordinate these processes. In the relax of this part, we will begin the two knowledge bases and each process in this workflow.

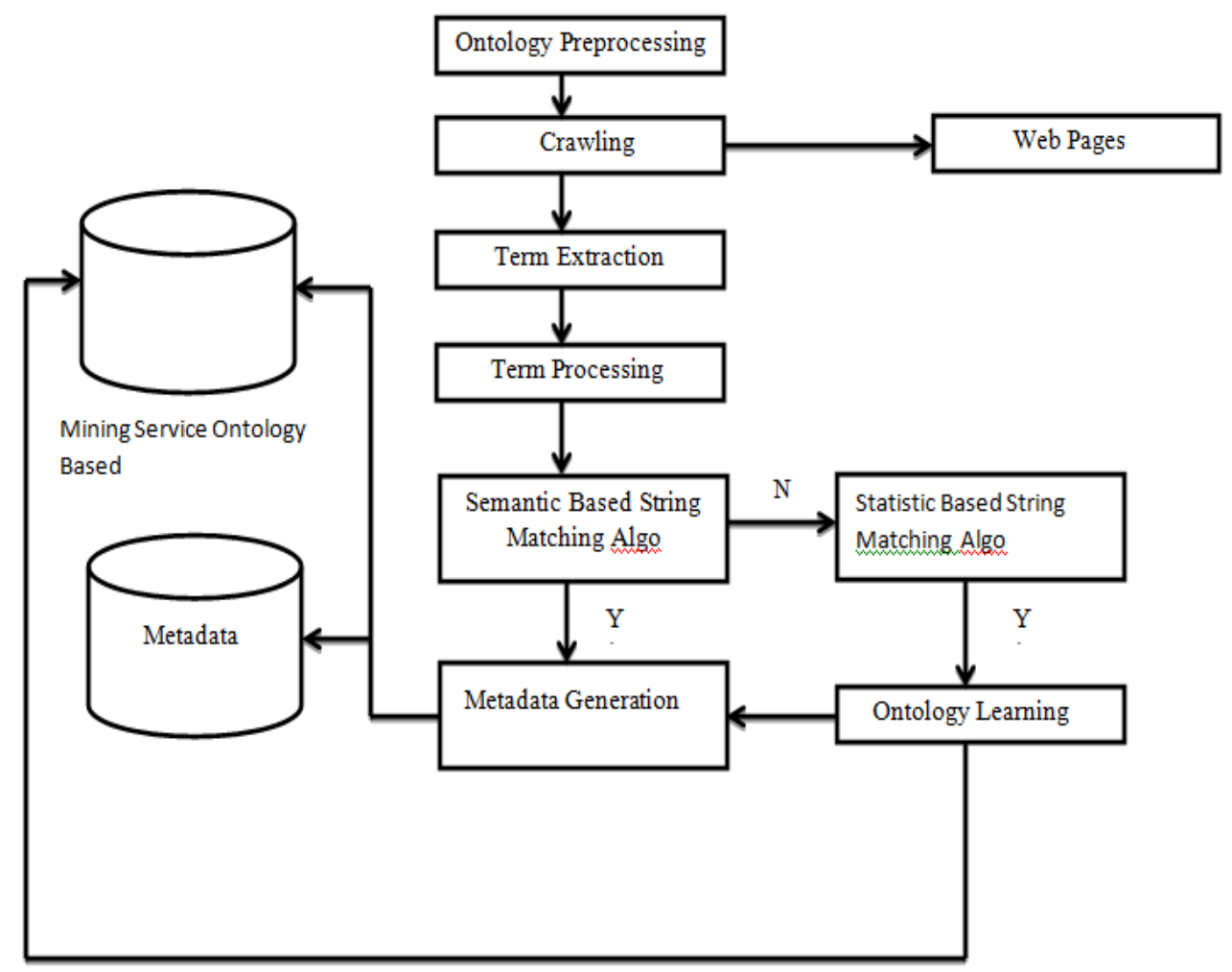

Fig: Self Adaptive Metadata and Ontology Learning

A. Mining Service Ontology Base and Mining Service Metadata Base

The Mining Service Ontology Base is used to accumulate mining service ontology, which is the demonstration of explicit mining service area information. Concept in the mining service ontology is ready in a hierarchical structure, and these concepts are connected by a overview/specialty connection, and are ordered in the form of a four-level hierarchy. Each idea in the mining check ontology represents a mining service sub-domain, and is defined by three properties - concept Description, learned Concept Description, and linked Metadata, which are expressed as follows:

- The concept Description property is a information form property second-hand to store up the textual descriptions of a mining service concept, which consists of numerous phrase in order to in brief summarize the categorize skin tone of the parallel mining service sub-domain. The 
- contents of each concept Description property are physically particular by domain expert and this will be used to compute the parallel value between a mining service idea and a mining service metadata.

- The learned Concept Description property is a data type property that has a purpose similar to that of the concept Description property. The difference between the two properties is that the former is automatically learned from Web documents by the SASF crawler.

- The linked Metadata property is an object property used to associate a mining service concept and semantically relevant mining check metadata. This possession is used to semantically index the generated mining service metadata by means of the concepts in the mining service ontology.

The Mining Service Metadata Base is used to store the repeatedly generate and indexed mining service metadata. Mining service metadata is the concept of an real mining service ad available in a Web document. The mining service metadata plan follows a arrangement related to the health service metadata plan defined in our earlier work, by which mining service metadata include two parts mining service provider metadata and mining service metadata. Mining service provider metadata is the concept of a service provider's profile, with the service provider's basic introduction, address, contact no, and so on. Mining service metadata has the properties of examine report and connected theory, which are declared as follows.

- The service explanation property is a datatype belongings, which contains the texts used to simplify the common features of an explicit service. The contents of this property are again and again removing from the mining service advertisements by the SASF crawler. This possession will be used for the later concept metadata connection computation.

The connected idea assets are the reverse property of the linked Metadata property, which stores the URIs of the semantically relevant mining service concept of removal repair metadata. It needs to be noted that mining service metadata and mining service concept is many-to- many relationship. In addition, mining service metadata and a related mining service provider metadata are associated by an object property of is Provided By, and this organization follows a many-to-one relationship because in detail, a service provider can provide more than one service.

\section{B. System Workflow}

In this section, we will introduce the system workflow of the SASF crawler step-by-step, as shown in Fig.

- The main goal of this crawler comprise:

1) To produce mining check metadata from Web pages

2) To precisely associate between the semantically related removal service concepts and mining service metadata with relatively low computing cost.

- The second goal is realized by:

1) measure the semantic relatedness among the concept explanation and learned Concept explanation property values of the concepts and the service explanation goods ideals of the metadata
2) Automatically learn new values, namely graphic phrase, for the learned Concept explanation properties of the concepts.

As can be seen in Fig the first step is preprocessing, which is to process the contents of the concept Description property of each concept in the ontology previous to similar the metadata and the concepts. This processing is realized by using Java Word Net Library8 (JWNL) to apply tokenization, part-of-speech (POS) tagging, nonsense word filtering, stemming, and synonym searching for the concept Description property values of the concepts. The second and third steps are crawling and word removal. The plan of these two process is to download Web pages from the Internet at one time, and to extract the necessary in sequence from the downloaded Web page, according to the mining service metadata schema and the mining service provider metadata schema defined in Section, in order to prepare the property values to create a new group of metadata.

The subsequently rung is word dispensation which is to practice the substance of the service Description possessions of the metadata in organize to set up for following model-metadata matching. The execution of this method is parallel to the completion of the preprocessing method. The main diversity is that period giving out does not require the purpose of synonym sharp for two most important reason: 1) the synonyms of the conditions in the idea- version property of concept have previously been retrieve in preprocessing; and 2) the compute rate of the synonym incisive for the conditions in the service Description property is quite towering and this may sway the scalability of the SASF crawler, as term processing is a real-time process. The relax of the workflow can be included as a self-adaptive metadata association and ontology learning process. The information of this procedure are as follows:

First of all, the direct string matching process examine whether or not the stuffing of the service Description property of metadata are integrated in the concept Description and learned Concept Description properties of a conception. If the answer is 'yes', then the insight and the metadata are regard as semantically related. in funds of metadata invention and involvement method, the metadata can then be generate and store in the insertion service metadata support as well as individual connected with the conception. If the react is 'no', an algorithm-based string matching process will be summon to prove the semantic relatedness among the metadata and the model, by capital of a model metadata semantic parallel algorithm (introduced in Section IV). If the conception and the metadata are semantically related, the stuffing of the service Description land of the metadata can be view as a clean cost for the learned Concept Description property of the conception. The metadata is thus allowable to go through the metadata generation and organization practice; if not the metadata is regard as semantically non-related to the perception. The over procedure is repeat awaiting all the concept in the mining service ontology have been compare with the metadata. If none of the concepts is semantically related to the metadata, this metadata is 
regard as semantically non-related to the mining service area and will be drop.

It wants to be noted that only the concept Description property morals of the concepts can be used in the algorithm-based string similar procedure, due to the fact that the semantic relatedness between the concept and the metadata is determined by comparing their algorithmbased property match values with a entrance price. If the highest match value between the service Description property value of a metadata and the concept Description property values of a concept is higher than the entrance price, the metadata and the concept are regarded as semantically related; otherwise not. Hence, the threshold value can be viewed as the edge for influential whether or not the service Description property value of the metadata is semantically relevant to a concept Description property value of a concept, and the concept Description property values of this concept can be viewed as the organization for constructing this border.

\section{CONCLUSION}

We presented a modern ontology learning based focused crawler - the SASF crawler, for service information find in the mining service trade, by taking into report the heterogeneous, ubiquitous and ambiguous life of mining service information vacant more than the Internet. This loom occupied an original unsupervised ontology learning support for terms-base ontology learning, and a new ideametadata matching algorithm, which combine a semanticsimilarity-based SeSM algorithm and a possibility-based StSM algorithm for associate semantically related mining service concepts and mining service metadata.

This loom enable the crawler to job in an uninhibited background where the many original vocabulary and ontologies worn by the crawler have a narrow array of glossary Then, we manner a cycle of experiment to empirically price the show of the SASF crawler, by comparing the show of this loom by the offered approaches based scheduled the six parameter adopt from the IR meadow We tell a control of this advance and our hope effort as follows: in the costing time, it can be visibly see that the concert of the self-adaptive form did not fully gather our hope about the parameter of accuracy and recollect. We assume two reasons that cause this copy as follows: initially, in this study, we seek to discover a common brink cost for the idea-metadata semantic similarity algorithm in sort to place positive a limit for formative idea-metadata relatedness. but, in array to reach best presentation all theory should have its personal fussy limitations specifically fussy brink ethics, for the result of the relatedness. therefore, in hope examine, we plan to propose a semi-supervised advance by aggregate the unsupervised advance and the supervised ontology learning-based advance with the reason of repeatedly choose the finest entry principles for every assumption, as trust the most select act lacking allowing for the drawback of the guidance.

Secondly, the related examine metaphors for every idea is automatically resolute during a examine-review course; i.e., several related examination metaphors and notion metaphors are determined on the origin of familiar intelligence, which cannot be judge by cord parallel or time co-occurrence. Hence, in our future study, it is basic to develop the dictionary of the mining service ontology by survey those matchless but related service images, in arrange to advance the act of the SASF crawler.

\section{ACKNOWLEDGMENT}

I thank my project guide Prof. Mohini J. Arote and BE. Coordinator Prof. B. H. Burghate for the guidelines in completion of this paper. I also wish to record my thanks to our Head of Department Prof. G. M. Bhandari for consistent encouragement and ideas

\section{REFERENCES}

1. H. Wang, M. K. O. Lee, and C. Wang, "Consumer privacy concerns about Internet marketing," Commun. ACM, vol. 41, pp. 63-70, 1998.

2. R. C. Judd, "The case for redefining services," J. Marketing, vol. 28, pp. 58-59, 1964.

3. T. P. Hill, "On goods and services," Rev. Income Wealth, vol. 23, pp. 315-38, 1977.

4. C. H. Lovelock, "Classifying services to gain strategic marketing insights,” J. Marketing, vol. 47, pp. 9-20, 1983.

5. H. Dong, F. K. Hussain, and E. Chang, "A service search engine for the industrial digital ecosystems," IEEE Trans. Ind. Electron., vol. 58, no. 6, pp. 2183-2196, Jun. 2011.

6. Mining Services in the US: Market Research Report IBISWorld2011.

7. B. Fabian, T. Ermakova, and C. Muller, "SHARDIS - A privacyenhanced discovery service for RFID-based product information," IEEE Trans. Ind. Informat., to be published.

8. H. L. Goh, K. K. Tan, S. Huang, and C. W. d. Silva, "Development of Bluewave: A wireless protocol for industrial automation," IEEE Trans. Ind. Informat., vol. 2, no. 4, pp. 221-230, Nov. 2006.

9. M. Ruta, F. Scioscia, E. D. Sciascio, and G. Loseto, "Semanticbased enhancement of ISO/IEC 14543-3 EIB/KNX standard for building automation," IEEE Trans. Ind. Informat., vol. 7, no. 4, pp. 731-739, Nov. 2011.

10. I.M.Delamer and J. L. M. Lastra, "Service-oriented architecture for distributed publish/subscribe middleware in electronics production,” IEEE Trans. Ind. Informat., vol. 2, no. 4, pp. 281-294, Nov. 2006.

11. H.Dong and F. K. Hussain, "Focused crawling for automatic service discovery, annotation, and classification in industrial digital ecosystems," IEEE Trans. Ind. Electron., vol. 58, no. 6, pp. $2106-$ 2116, Jun. 2011.

12. H. Dong, F. K. Hussain, and E. Chang, "A framework for discovering and classifying ubiquitous services in digital health ecosystems," J. Comput. Syst. Sci., vol. 77, pp. 687-704, 2011. 